\title{
OPTIMIZED ANTENNAS FOR MOBILE COMMUNICATION BASE STATIONS
}

\author{
J.R. Sanford ${ }^{1}$, J.- F. Zürcher ${ }^{2}$ and S. Robert ${ }^{3}$
}

\section{ABSTRACT}

This is a synopsis of work conducted to improve the electrical and mechanical performance of antennas used for mobile communication base stations. To accomplish this we first consider the propagation characteristics to determine the optimal antenna pattern. In addition, the antennas mechanical size and profile are minimized to improve its appearance and mounting ease. The end result of this work is an antenna that has superior performance in terms of virtually every antenna and mechanical specification. This was achieved by the use of a specially constructed microstrip patch element in conjunction with technology for beam shaping and the use of environmentally proven materials. The present use for such antennas is for the $900 \mathrm{MHz}$ GSM and $1.8 \mathrm{GHz}$ PCN systems base stations but freedom in the design frequency range, polarization, and array configurations allow for a considerably wider range of applications.

\section{PATTERN REQUIREMENTS}

The specification of an antenna used for mobile communications has primarily the same desired characteristics as antennas used point to point communications with the exception that it must illuminate an area rather than provide extremely high gain in one particular direction. The propagation characteristics are effected by many factors including the frequency, distance, antenna heights, curvature of the earth, atmospheric conditions, and the presence of hills and buildings. Typically antennas will not be designed for individual cases but rather for the most common occurring cases thus in the design of the antenna one considers the parameters which remain constant and the averages the parameters which significantly vary. The presence of the ground modifies the generation and the propagation of radio waves so that the received field intensity is ordinarily less than would be expected in free space. The ground acts as a partial reflector and as a partial absorber, and both of these properties affect the distribution of energy in the region above the earth. The principle effect of plane earth on the propagation of radio waves is indicated by the following equation ${ }^{\text {[I] }}$

$$
E-E_{0}\left[1+R e^{j 8}+(1-R) A e^{j 8}+\ldots\right]
$$

where the equation is the sum of a direct wave, reflected wave, surface wave, plus the induction field and secondary effects of the ground. The angle $\delta$ is the phase difference in radians resulting from the difference in the length of the direct and reflected rays. The reflection coefficient of the ground, $R$, is equal to -1 when the angle $\Theta$ between the reflected

\footnotetext{
1 Huber \& Suhner AG, 9100 Herisau, Switzerland; 2 LEMA, Ecole Polytechnique Fédérale de Lausanne, Lausanne, Switzerland; 3 DME, Ecole Polytechnique Fédérale de Lausanne, Lausanne, Switzerland.
} 
ray and the ground is small. For a vertically polarized wave in general

$$
R=\frac{\sin \theta-z}{\sin \theta+z}
$$

where

$$
z=\sqrt{\frac{e-j 60 \sigma \lambda-\cos ^{2} \theta}{e-j 60 \sigma \lambda}}
$$

and

$$
\begin{aligned}
& \text { e-relative dielectric constant of ground } \\
& \text { o-ground conductivity (mhos/meter) } \\
& \lambda-\text { wavelength (meters) }
\end{aligned}
$$

The surface-wave attenuation factor $A$ depends upon the frequency, ground constants, and type of polarization. It is never greater than unity and decreases with increasing distance and frequency as indicated by the following approximation.

$$
A \sim \frac{-1}{1+j \frac{2 \pi r}{\lambda}(\sin \theta+z)^{2}}
$$

This approximation is sufficiently accurate as long as $A<0.1$, and gives the magnitude of $\mathrm{A}$ within about $2 \mathrm{~dB}$ for all values of $A^{[2]}$.

The optimal pattern for cellular radio is one which delivers approximately a constant maximized power to the contour of single cell and as little as possible to other cells. This is determined by considering the terrain type, antenna height and cell size. A system designer may want to generate a particular radiation pattern in which case shaped beam antennas are the preferred solution. One example is the $\csc ^{2} \boldsymbol{\theta}$ antenna. Consider an antenna with a power distribution $P(\Theta, \phi)$, mounted on a mast of known height $h_{1}$, above a flat terrain with known electrical properties. If the signal receivers are atHx constant height $h_{2}$ above the ground, as shown in figure 2, with the separation is given by $r$, then elevation given by $\Theta$ is given by

$$
\csc \theta-\frac{r}{(h 1-h 2)}
$$

If the receivers are all in the half-plane defined by $\phi=0^{\circ}$, then the received power at a given receiver is

$$
E_{0} \sim \sqrt{\frac{G, P\left(\theta, 0^{\circ}\right)}{r^{2}}}
$$




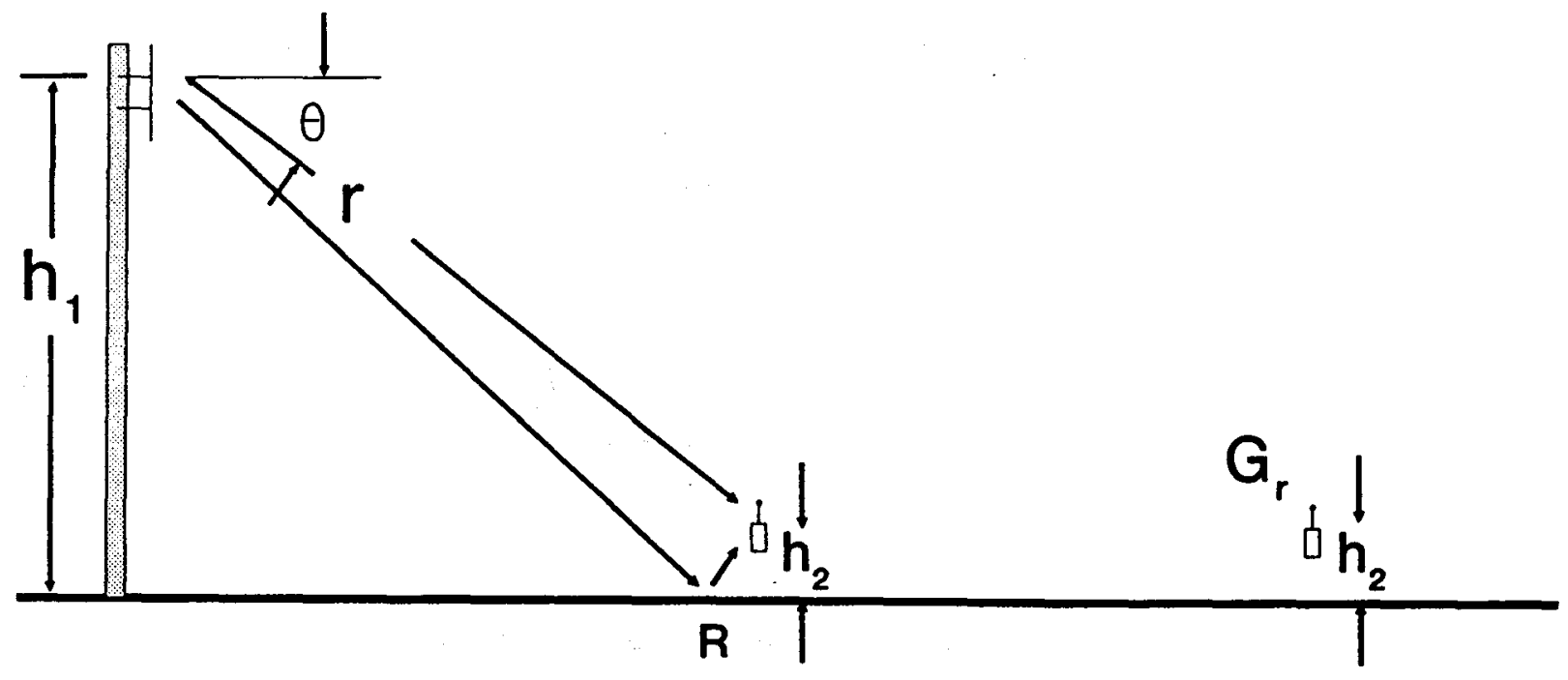

Figure 1. User-relay configuration on a flat earth

where $G_{r}$ is the gain of the receiving antenna. It is desired to have the received power equal at all positions, thus

$$
\frac{P\left(\theta, 0^{\circ}\right)}{r^{2}} \sim \text { constant }
$$

When equations (5) and (7) are combined we find

$$
P\left(\theta, 0^{\circ}\right) \sim \csc ^{2} \theta
$$

Thus if the principal plane pattern is designed to be proportional to the square of cosecant of the elevation angle, the direct radiation at a constant elevation over a flat earth will be approximately a constant independent of the ground reflection. Modifications of the $\csc ^{2} \theta$ formula are obtained when assumptions are made about the ground reflections and land contour.

The beam shaping technique used in most of these antenna is a Fourier method ${ }^{[3]}$ which allows an arbitrary pattern function to be approximated with minimum RMS error using a finite number of radiating elements. The Woodward method ${ }^{[4]}$ is used when specific pattern nulls are needed. The quality of the pattern approximations increases with the number of array elements and thus the size of the antenna but significant shaping can be done with as little as 7 elements. In contrast conventional broadside antennas typically have many nulls where no link can be made while supplying a higher power to a relatively small area.

Energy can be transmitted beyond the horizon into additional cells by atmospheric scattering or through the back lobes of the antenna. Therefore the upper hemisphere sidelobes should be minimized along with maximizing the front-to-back ratio. In addition interference between cells occurs through direct and scattered radiation from the main lobe. Since the detrimental effects of both occur at angles very near the horizon the antenna can not discriminate between the cells and thus no optimization can be done. 


\section{PATCH ELEMENT TECHNOLOGY}

Microstrip patch elements are low weight, low profile and small in size. They work extremely well in the array environment due to their low mutual coupling characteristics. Their rugged construction allows deployment in the worst of environmental conditions while the configuration makes them easy to mount and visually acceptable. Such elements are commonly used for frequencies from $1 \mathrm{GHz}$ to $25 \mathrm{GHz}$. The only drawback of patch elements until now has been their limited bandwidth. This problem has been resolved by the use of multiple layer slot fed patches which can yield bandwidths in excess of 30\% patch configuration also lends itself well to a combination with a microstrip feeding network, which can generate complex phase and amplitude tapers allowing a maximum degree of freedom for beam shaping.

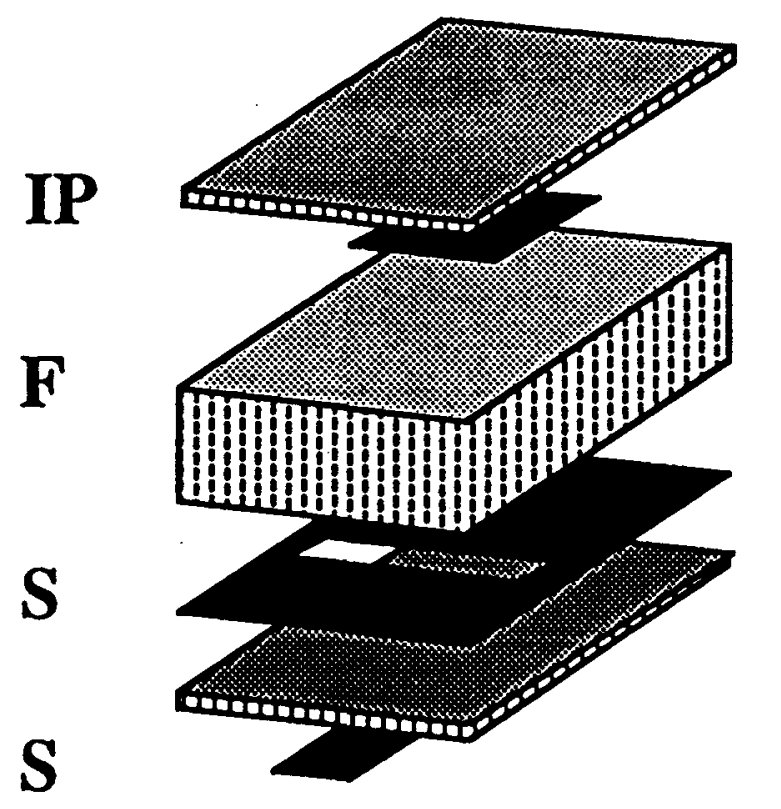

thin dielectric (top cover) with patch printed on underside inverted radiating patch

FOAM

ground plane with slot

microstrip substrate

microstrip line (feed) with quarter wave stub

Figure 2. Exploded view of SSFIP element

The strip slot foam inverted patch (SSFIP) shown in figure 1 provides bandwidths around $13 \%$ while being extremely durable due to the integrated radome. Both glass and epoxy composites have been employed as radome materials. The choice of common building materials such as these guarantees that the antenna is environmentally protected. Designs have been done with both linear and circular polarizations. The gain of such elements is about $8 \mathrm{~dB}$ while the cross polar levels are typically better than $25 \mathrm{~dB}$.

\section{FEED NETWORKING AND INTEGRATION}

The feed network has been etched on a low loss dielectric board using microstrip technology ${ }^{[7]}$. It takes the form of a corporate feed with an uneven phase and power 
optimal beam shaping capability. The network employs symmetrical and asymmetrical Wilkinson power dividers which have been test until 100 Watts. The microstrip lines are terminated in quarter wave stubs at which point the power is coupled through the elements slot and radiated. This configuration allows the network to reach bandwidths in excess of the element's bandwidth, thus the overall bandwidth of the system increases about $20 \%$ due to the cancellations of element reflections not adding in phase.

Each of the components is fabricated by a photolithographic or similar procedure. Due to the lack of mechanical connection between planes the SSFIP concepts requires no costly drilling and hole plating. In addition, this method eliminates the need for mechanical connections and thus provides an antenna with as little intermodulation as possible. All the materials used are quite lightweight and relatively inexpensive resulting in a price competitive antenna that is easy to deploy. An example of such an antenna and its characteristics are shown in figure 3 which is translated into incident power as a function of range in figure 4.

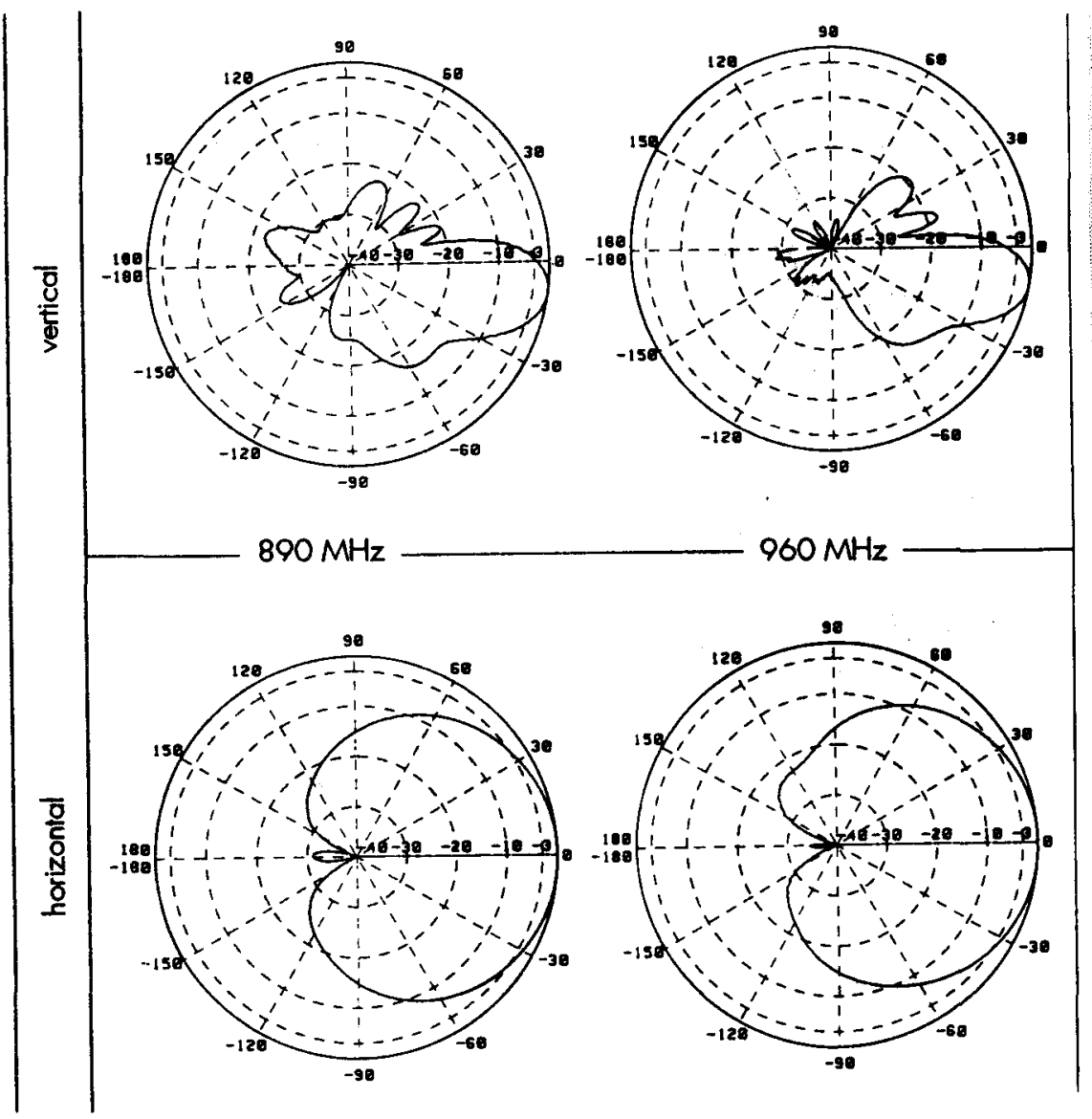

RETURN LOSS (dB)
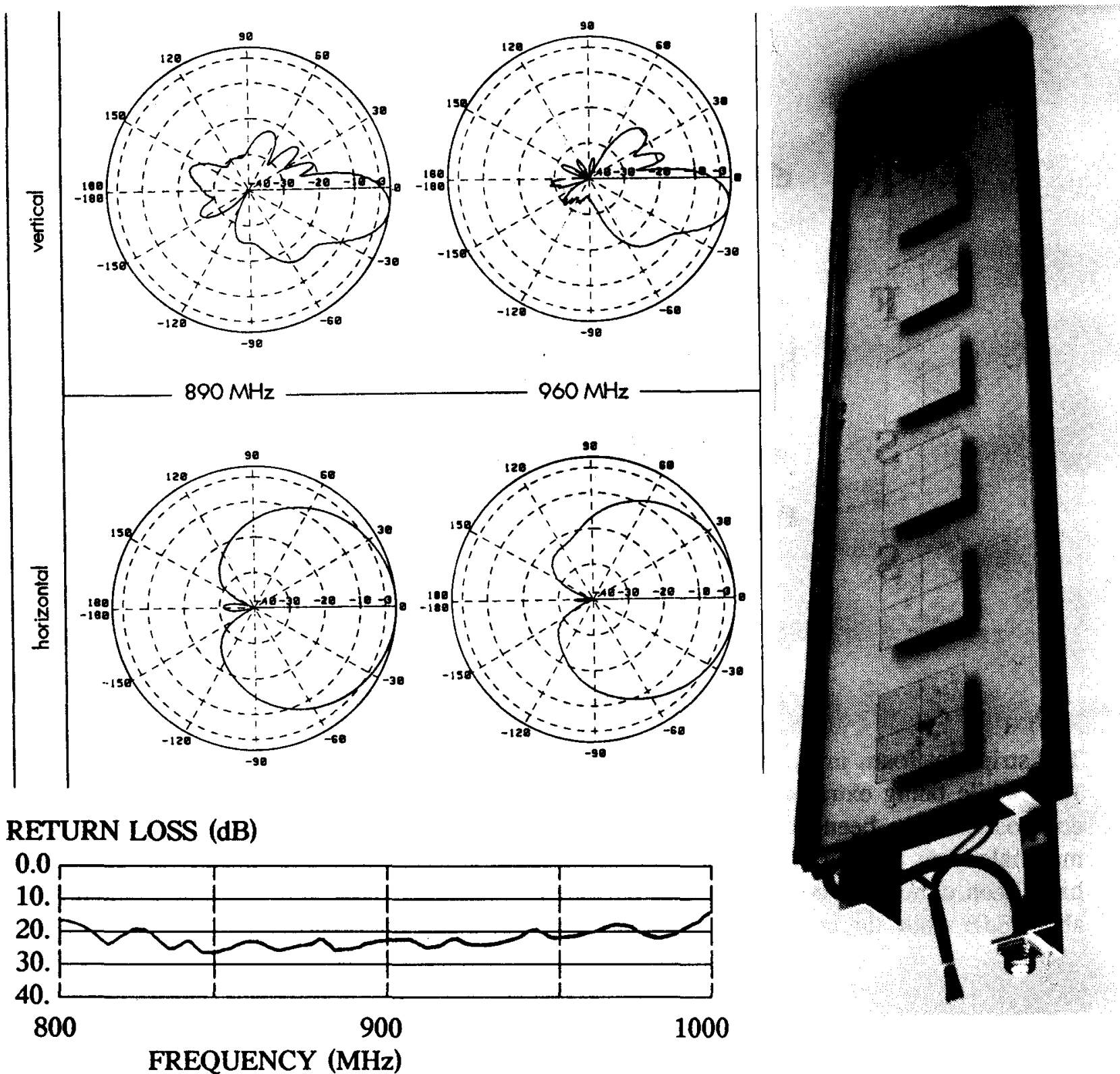

Figure 3 - Antenna and Specifications 


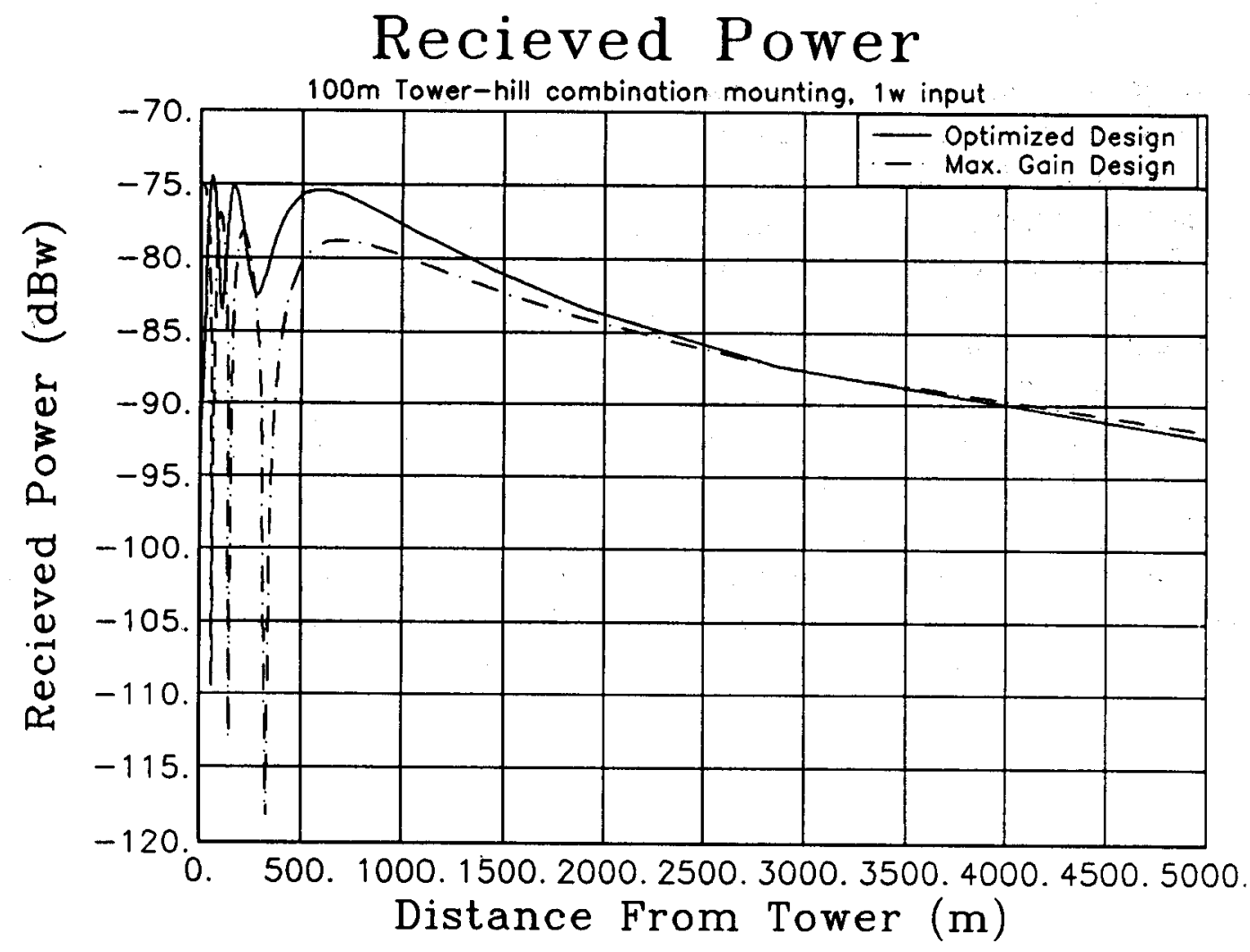

Figure 4. Incident power as a function of range

The complex phasing of the array elements allows the required beam tilt to be directly integrated into the antenna rather than adding a mechanical beam tilt. The same technique can be applied to a set of vertical columns of element to create a fixed tilt in azimuth. This capability allows the integration of the antenna into fixed building walls, still allowing the beam to be pointed in a given range of directions.

Such a design has advantages over conventional dipole antennas in that it can be coorperatly fed and thus has virtually no beam skewing with frequency. Due to the difficulties in feeding dipole arrays it is quite difficult to generate a pattern with no nulls let alone a pattern with significant shaping. In addition, the patch array need only be about a seventh of a wavelength thick while a dipole array must be significantly more than a quarter wavelength. The major draw back in the path technology are the difficulties in generating patterns with $\mathrm{H}$-plane $3 \mathrm{~dB}$ beamwidths greater $80^{\circ}$.

\section{CONCLUSION}

The antenna design presented in this paper has seemingly only advantages over conventional antennas for mobile communication base stations in term of both its electrical and mechanical specifications. The improvement comes from a combination of broad band patch technology in conjunction with beam shaping techniques. Should one desire, the antenna can be designed to yield the same pattern characteristics of the standard dipole array presently used but typically will be designed to exceed such specifications. 


\section{REFERENCES}

[1] K. Bullington, "Radio Propagation for Vehicular Communications," IEEE Trans. on vehicular technology, vol. VT-26, No 4, Nov 1977

[2] K.A. Norton, "The calculation of ground-wave field intensities over a finitely conduction spherical earth," Proc. I.R.E., vol. 29, pp. 623-639

[3] C.A. Balanis, "Antenna Theory," Harper \& Row, 1982

[4] R.C. Hansen (Ed.), "Microwave Scanning Antennas," Academic Press Inc., vol 1, 1964, vol 2, 3, 1966.

[5] J.-F. Zürcher,"The SSFIP - A global concept for high performance broadband antennas," Electronics Letters, vol. No 23, pp. 1433-1435, Nov. 1988

[6] B. Zürcher, J.-F. Zürcher and F.E. Gardiol: "Broadband Microstrip Radiators - The SSFIP concept", Electronics 9, pp. 385-393, 1989

[7] J.-F. Zürcher,"MICROS3 - A CAD/CAM program for the fast realization of microstrip masks, description and typical applications," IEE Coll. on Computer-Aided Design of Microwave Circuits, London, November 1985, pp. 11/1-5 